\title{
Simple, Fast, and Cost-Effective Fabrication of Wafer-Scale Nanohole Arrays on Silicon for Antireflection
}

\author{
Di Di, ${ }^{1}$ Xuezhong Wu, ${ }^{1}$ Peitao Dong, ${ }^{1,2}$ Chaoguang Wang, ${ }^{1}$ Jian Chen, ${ }^{1}$ \\ Haoxu Wang, ${ }^{1}$ Junfeng Wang, ${ }^{1}$ and Shengyi $\mathrm{Li}^{1}$ \\ ${ }^{1}$ College of Mechatronics and Automation, National University of Defense Technology, Changsha 410073, China \\ ${ }^{2}$ State Key Laboratory of Transducer Technology, Chinese Academy of Sciences, Shanghai 200050, China \\ Correspondence should be addressed to Peitao Dong; ptdong@nudt.edu.cn
}

Received 12 February 2014; Accepted 21 April 2014; Published 15 June 2014

Academic Editor: Jie-Fang Zhu

Copyright (C) 2014 Di Di et al. This is an open access article distributed under the Creative Commons Attribution License, which permits unrestricted use, distribution, and reproduction in any medium, provided the original work is properly cited.

A simple, fast, and cost-effective method was developed in this paper for the high-throughput fabrication of nanohole arrays on silicon ( $\mathrm{Si}$ ), which is utilized for antireflection. Wafer-scale polystyrene (PS) monolayer colloidal crystal was developed as templates by spin-coating method. Metallic shadow mask was prepared by lifting off the oxygen etched PS beads from the deposited chromium film. Nanohole arrays were fabricated by Si dry etching. A series of nanohole arrays were fabricated with the similar diameter but with different depth. It is found that the maximum depth of the Si-hole was determined by the diameter of the Cr-mask. The antireflection ability of these Si-hole arrays was investigated. The results show that the reflection decreases with the depth of the Si-hole. The deepest Si-hole arrays show the best antireflection ability (reflection $<9 \%)$ at long wavelengths $(>600 \mathrm{~nm}$ ), which was about 28 percent of the nonpatterned silicon wafer's reflection. The proposed method has the potential for high-throughput fabrication of patterned Si wafer, and the low reflectivity allows the application of these wafers in crystalline silicon solar cells.

\section{Introduction}

Solar cell is an important development direction for clean and renewable energy [1]. Crystal silicon solar cells dominated a significant position in the commercial solar cell market [2]. However, high reflectivity of silicon wafer on visible light (more than 30\%) has greatly hindered the light conversion efficiency $[2,3]$. Recently, various antireflection methods have been developed to reduce the reflection loss of solar cells, such as antireflection multilayer films and patterned coatings [4].

Constructing periodic patterns directly on silicon substrates is attractive for solar cells due to its strong enhanced light trapping and potential low cost [4]. For example, vertically aligned nanowire, nanorod, nanocone, or nanohole arrays have been studied to improve light absorption for solar cells [4-9]. Theoretical research suggests that nanohole architectures display strong optical absorption ability because of the effective optical coupling between the array and the incident sunlight as well as the large density of waveguide modes [4]. Nanohole arrays also exhibit better mechanical robustness, compared with multilayer antireflection films and free-standing nanowire structures.

As mentioned above, nanohole arrays have great potential for efficient solar photovoltaics. A simple, controllable, costeffective, and high-throughput fabrication method was of primary importance for practical commercial application. Deep ultraviolet lithography and electron beam lithography have been used to fabricate the nanopatterns on silicon wafer surface $[4,10]$. However, the high cost and lowthroughput hindered its wide application. Laser holographic lithography has also been used to fabricate silicon nanoholes [11-13]. It was considered a potentially high throughput process for periodic nanostructures fabrication. However, it is limited by the light wavelength in fabrication and the huge cost. Nanosphere lithography has been widely used in the fabrication of periodic nanopatterns for its low cost and good controllability [14-19]. Manufacturing efficiency may be the biggest problem commonly encountered $[2,3,9]$. 


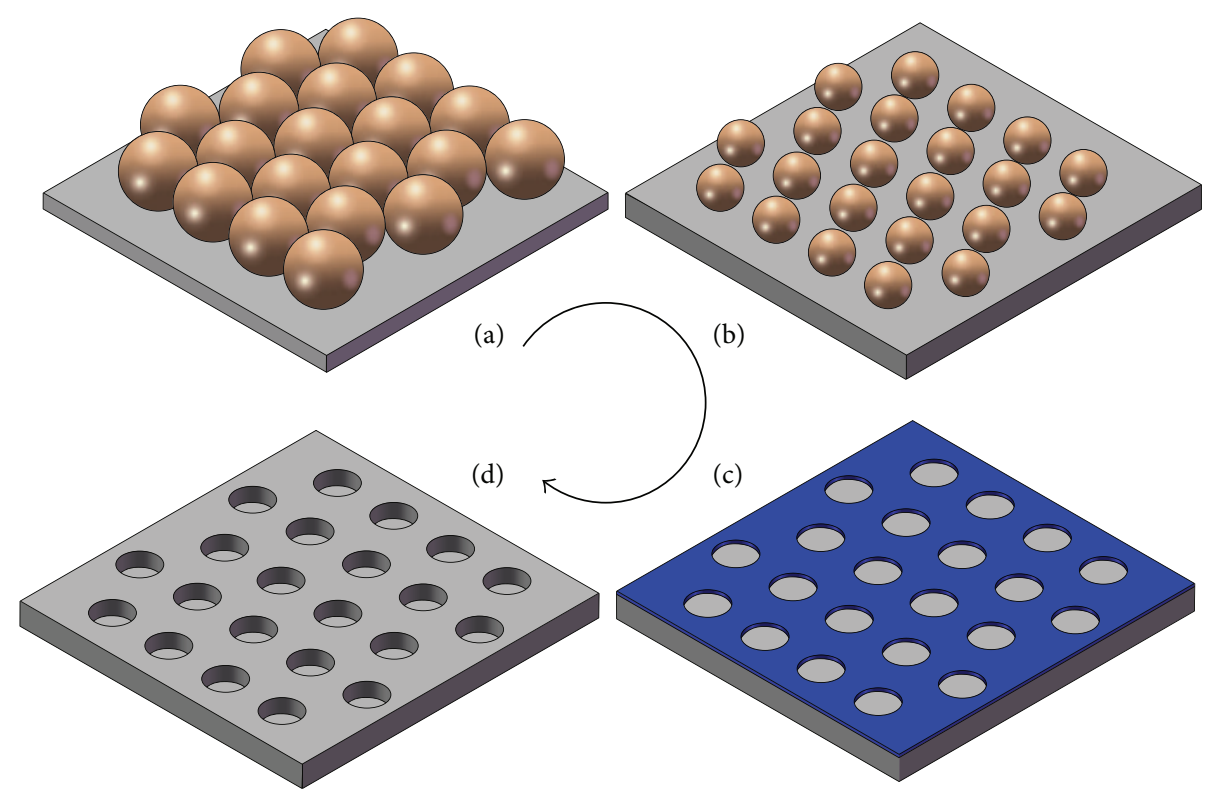

FIGURE 1: Schematic of nanohole array fabrication procedures: (a) PS monolayer colloidal crystal prepared by spin-coating; (b) PS beads size reduction by oxygen etching; (c) Si-hole preparation by lifting off the etched PS bead from the Cr-layer; (d) Si-hole array fabrication by silicon dry etching.

Herein, we demonstrated a simple, fast, and costeffective strategy for high-throughput fabrication of antireflective nanohole arrays on silicon. Spin-coating method was employed to fabricate wafer-scale crystal templates. And some other standard microfabrication methods, such as $\mathrm{O}_{2}$ reaction ion etching, metal film deposition, and silicon dry etching, were in turn implemented to fabricate silicon nanoholes arrays. A series of Si-hole arrays were successfully fabricated in a controlled manner. And their antireflection ability was further investigated.

\section{Experimental}

2.1. Materials and Instruments. PS beads with a mean diameter of $310 \mathrm{~nm}$ were purchased from Sigma-Aldrich (Germany). $\langle 100\rangle$ silicon wafers $(500 \mu \mathrm{m}$ thickness, $\mathrm{n}$ type, from Tianjin Semiconductor Technology Research Institute) were used as substrates. Other chemicals were purchased from Sinopharm (Shanghai), including concentrated sulfuric acid $\left(\mathrm{H}_{2} \mathrm{SO}_{4}, 98 \%\right)$, hydrogen peroxide $\left(\mathrm{H}_{2} \mathrm{O}_{2}, 30 \%\right)$, ethanol $(\mathrm{EtOH})$, and acetone. $\mathrm{Cr}$ etchants and deionized water (DI) were prepared in laboratory. All chemicals were used as received.

A spin-coater (EASYLINE-S-200TT, Solar-semi, Germany) was employed to prepare well-organized crystal domain. An inductively coupled plasma reactive ion etching system (ICP-2B, Chuangweina, Beijing) was utilized to etch the PS beads and Si substrate. An e-beam evaporator (ZZS500, Nanguang, Chengdu) was used for Cr-layer deposition. Optical microscopy, scanning electron microscopy (SEM, S-4800, Hitachi), and atomic force microscopy (AFM, dimension Icon, Bruker Nano Surfaces Division) were used to analyze the nanoscale morphologies. UV-VIS spectrophotometer (Hitachi U-4100) was used here for reflectivity measurements.
2.2. Fabrication Procedures. The schematic of nanohole array fabrication procedures was illustrated in Figure 1.

PS beads were first dispersed in a mixed solution of $\mathrm{H}_{2} \mathrm{O}$ and $\mathrm{EtOH}(\mathrm{v}: \mathrm{v}=1: 1)$. The silicon wafers were cleaned in the "Piranha" solution ( $\mathrm{v}: \mathrm{v}=3: 1$ mixture of $\mathrm{H}_{2} \mathrm{SO}_{4}$ and $\mathrm{H}_{2} \mathrm{O}_{2}$ ) for one hour to improve their hydrophilicity. The cleaned wafer was stored in ethanol and dried with a stream of nitrogen gas before use. A drop $(1200 \mu \mathrm{L})$ of PS dispersions was spread onto silicon wafer, and the solution was allowed to uniformly cover the wafer surface for $30 \mathrm{~s}$ before spincoating process started. The rotation speed was $2000 \mathrm{rpm}$ and spinning time was $120 \mathrm{~s}$. PS monolayer colloidal crystal was developed on the 3-inch silicon wafer and used as starting templates (Figure 1(a)).

The PS monolayer colloidal crystal was then etched in the oxygen plasma to reduce size, operating at $4.6 \mathrm{e}^{-1} \mathrm{~Pa}$ (chamber pressure), $32 \mathrm{sccm}$ (flow rate), and $28 \mathrm{~W}$ (RF power) (Figure 1(b)). Subsequently, a $200 \AA$ thick Cr film was deposited in an e-beam evaporator with the evaporation rate of $2 \sim 5 \AA / s$. The etched PS beads were then lifted off from the Cr-layer, leaving behind the Cr-hole mask (Figure 1(c)). Silicon substrate dry etching process was employed to fabricate the Si-hole arrays. A mixture of $\mathrm{SF}_{6}$ gas and $\mathrm{Ar}_{2}$ gas was used as etching gas with the operating parameter characters of $0.1 \mathrm{~Pa}$ (chamber pressure), $46 \mathrm{sccm}$ (flow rate), and $45 \mathrm{~W}$ (RF power). Cr nanohole mask was then removed by the $\mathrm{Cr}$ etchant as shown in Figure 1(d). Finally, silicon substrate was ultrasonically cleaned in deionized water for several minutes.

\section{Results and Discussions}

3.1. Wafer-Scale Fabrication. Photovoltaic production calls for simple, fast, and cost-effective fabrication process. Also, the process should be compatible with current techniques. 


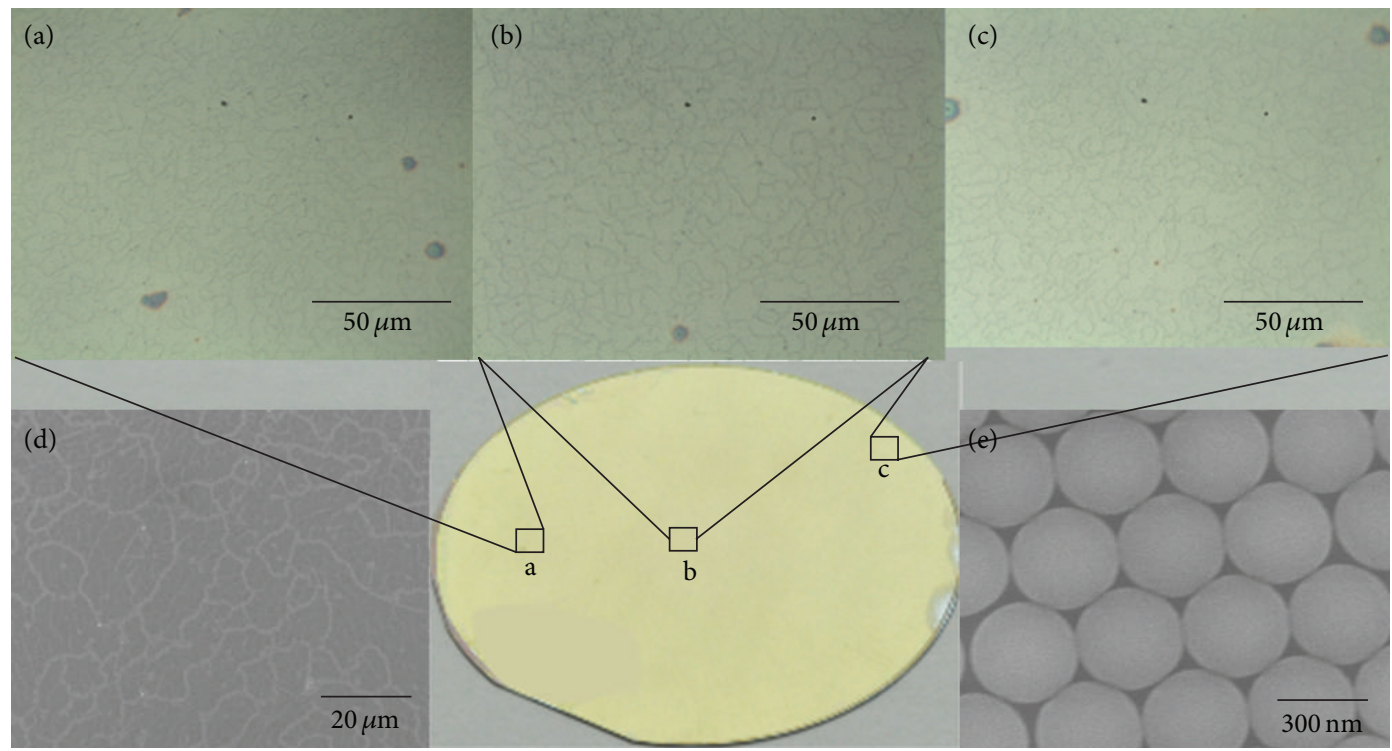

FIGURE 2: Optical microscopy photographs and SEM images of spin-coated PS monolayer. These points were randomly selected and kept a great distance away from each other.

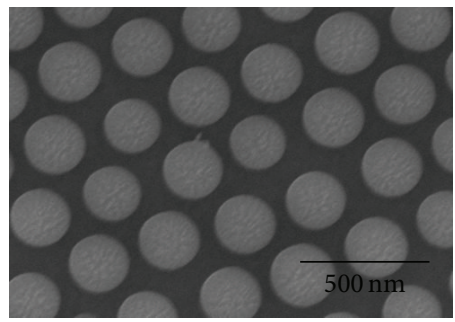

(a)

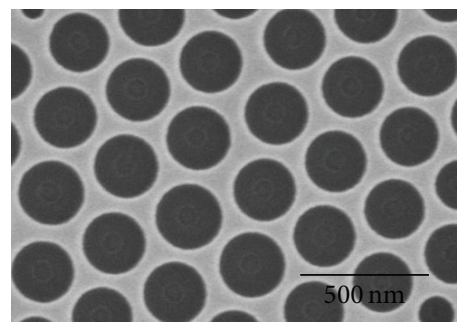

(d)

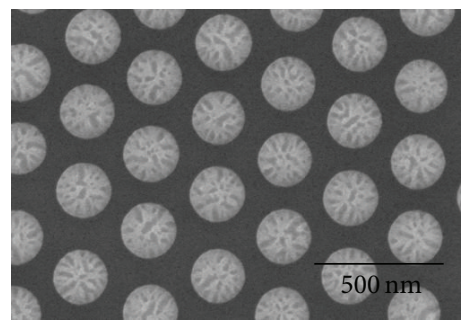

(b)

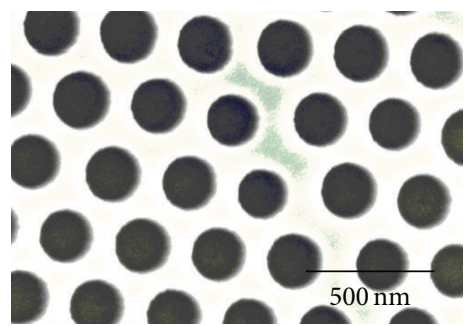

(e)

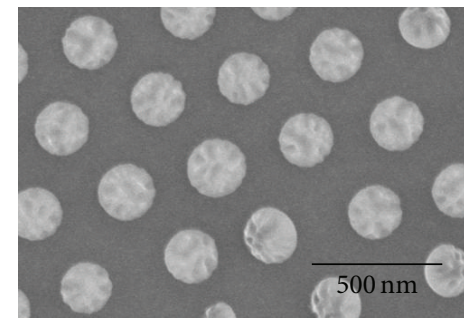

(c)

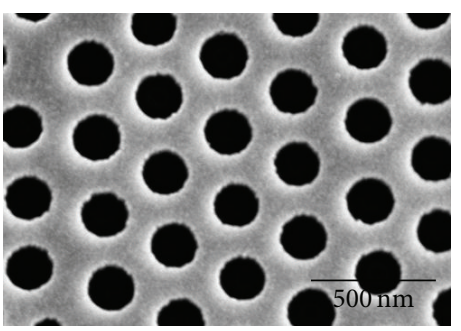

(f)

FIGURE 3: SEM images of size reduced PS NCP beads and the Cr nanoholes. ((a)-(c)) SEM images of PS beads with average diameters of $235 \mathrm{~nm}, 215 \mathrm{~nm}$, and $185 \mathrm{~nm}$, respectively; ((e)-(f)) SEM images of Cr nanoholes with average diameters of $244 \mathrm{~nm}, 219 \mathrm{~nm}$, and $191 \mathrm{~nm}$, respectively.

The nowadays widely used methods are either time-assuming or of low-throughput. Herein, spin-coating technique was utilized to prepare wafer-scale crystal domain. Spin-coating is a facile, cost-effective, and laborsaving technique, which is compatible with mature IC technique [20, 21].

Figure 2 shows a digital camera photograph of a 3inch silicon wafer, on which PS beads formed a uniform monolayer by spin-coating technique. Nearly the whole wafer presents yellow color when illuminated with white light. As the color of PS film changed obviously with the layer and the orientation of colloidal crystal, the homogeneity color of the wafer demonstrates the uniformity of the PS film [21]. The uniformity of PS film was further validated by the microscope images as shown in Figures 2(a), 2(b), and 2(c). The microscopy photographs were obtained at randomly selected areas that are far away from each other. It is monotonous yellowish green in the microscopy images. The arrays of close-packed PS beads were filled in the field of vision. The dark dots among the PS monolayer are a result of the incomplete dispersion of the PS sol., which can be avoided by complete dispersion.

Figures 2(d) and 2(e) show the low and high magnified SEM images of the PS monolayer colloidal crystal. The PS monolayer is constituted with regions of hexagonally 


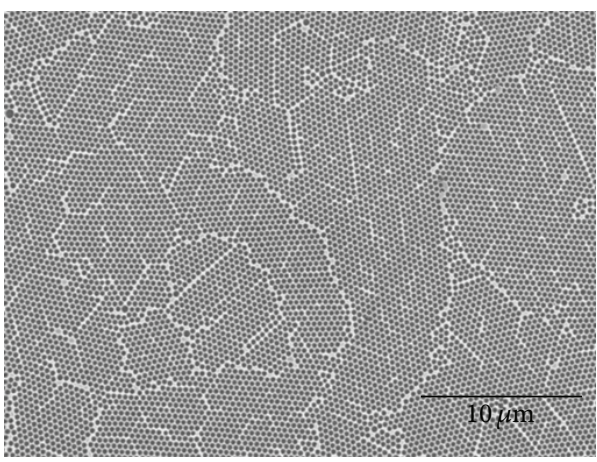

(a)

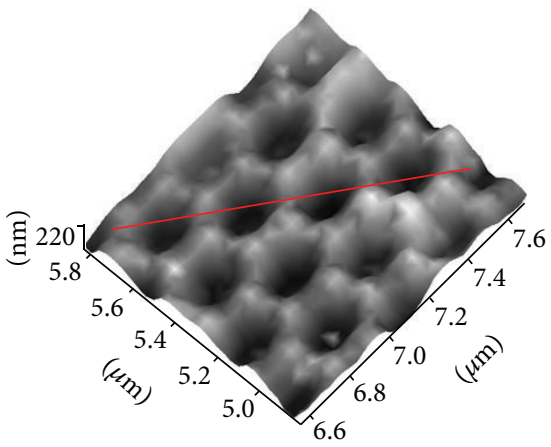

(c)

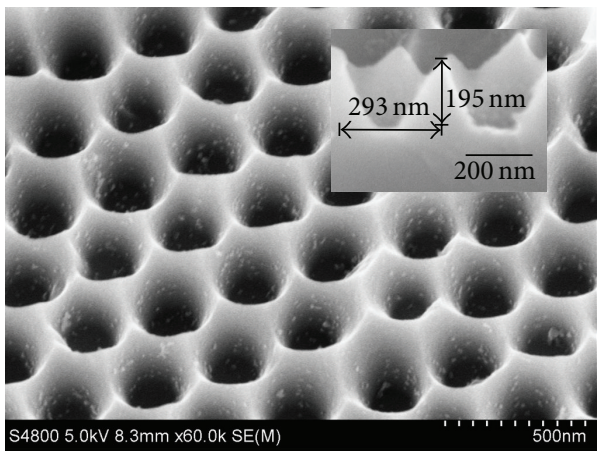

(e)

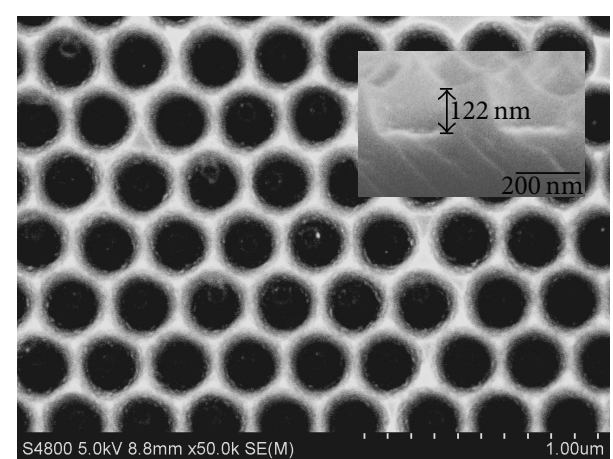

(b)

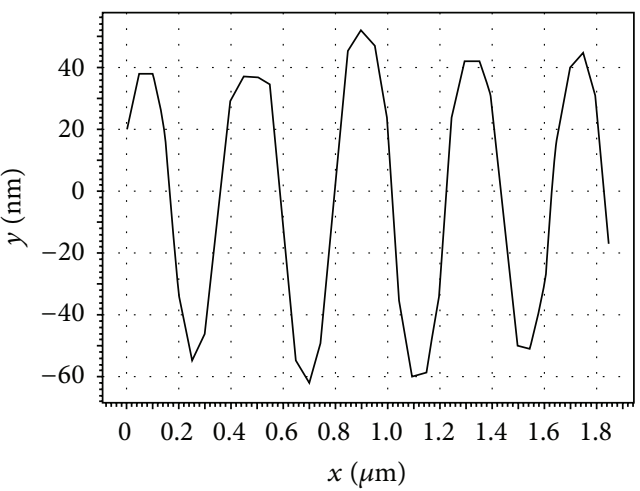

(d)

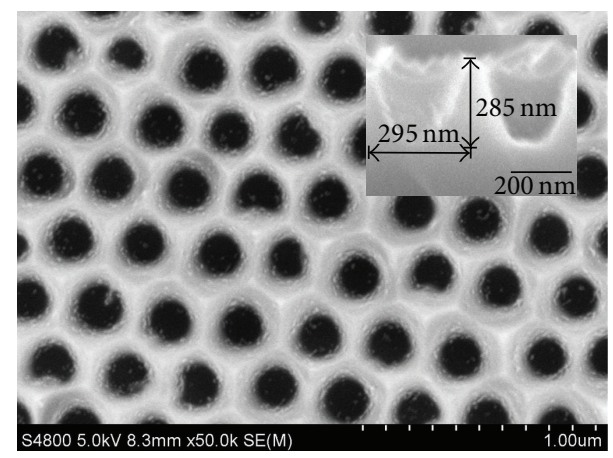

(f)

FIGURE 4: SEM and AFM images of Si-hole arrays. ((a)-(b)) The high and low magnification SEM images of Si-hole arrays after 3 min etching; (c) the 3D AFM images 3 min etched Si-hole arrays; (d) height profile of the red line marked in (c); ((e)-(f)) SEM images of Si-hole arrays after $5 \mathrm{~min}$ and $7 \mathrm{~min}$ etching, respectively.

close-packed (HCP) PS beads. Thus, the colloid film was long-range ordered. It has been demonstrated that there is no difference in photovoltaics for long-range ordered arrays and strict-ordered arrays [22]. The area of the HCP regions was on the order of several $1000 \mu \mathrm{m}^{2}$.

3.2. Etching Mask Preparation. To control the diameter of $\mathrm{Cr}$ mask, the PS beads were etched in $\mathrm{O}_{2}$ plasma. The oxygen etching parameters, such as etching power and etching time, were optimized to control the diameter of the PS beads. Figures 3(a), 3(b), and 3(c) show the SEM images of etched PS beads. The etched PS beads were nonclosed, packed, and round in shape seen from the top view. The beads' diameters were reduced to $235 \mathrm{~nm}, 215 \mathrm{~nm}$, and $185 \mathrm{~nm}$ after
$240 \mathrm{~s}, 260 \mathrm{~s}$, and $300 \mathrm{~s}$ oxygen etching, respectively. These nonclosed packed PS beads arrays were covered by Cr-layer and then lifted off by adhesive tape leaving behind the $\mathrm{Cr}$-hole arrays as shown in Figures 3(d), 3(e), and 3(f). The Cr-hole arrays served as etching mask in silicon nanohole fabrication. The diameters of Cr-hole are $244 \mathrm{~nm}, 219 \mathrm{~nm}$, and $191 \mathrm{~nm}$, respectively. They are a little larger than corresponding colloidal templates, which is a result of the lateral growth of the deposited metal [23].

3.3. Vertical Etching and Lateral Erosion. To investigate the relationship between the antireflection ability and the depth of the Si-hole, the diameters of Si-holes were controlled to about $300 \mathrm{~nm}$, which is much close to the period of 


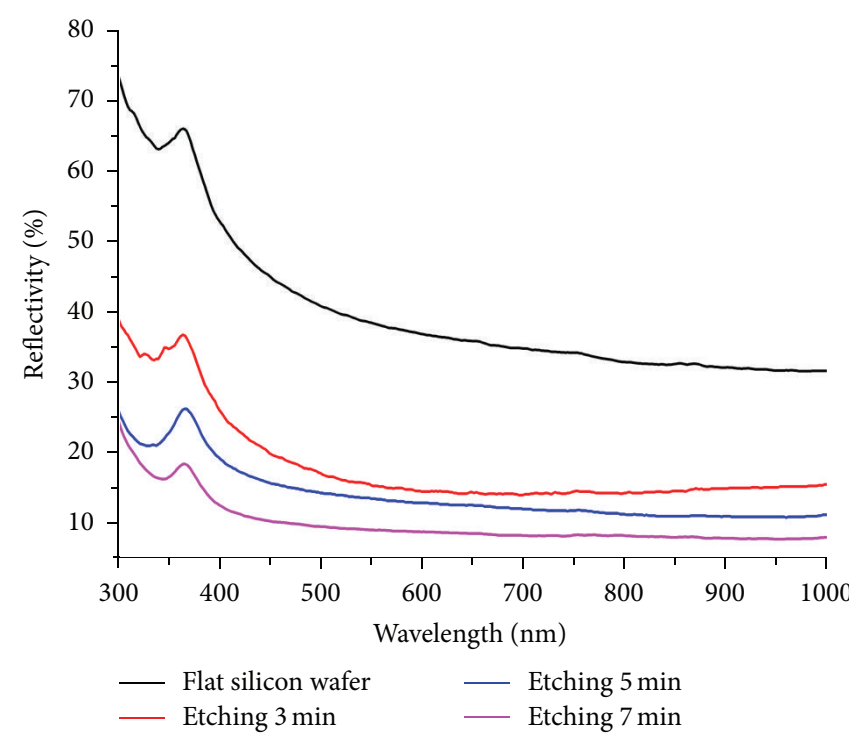

(a)
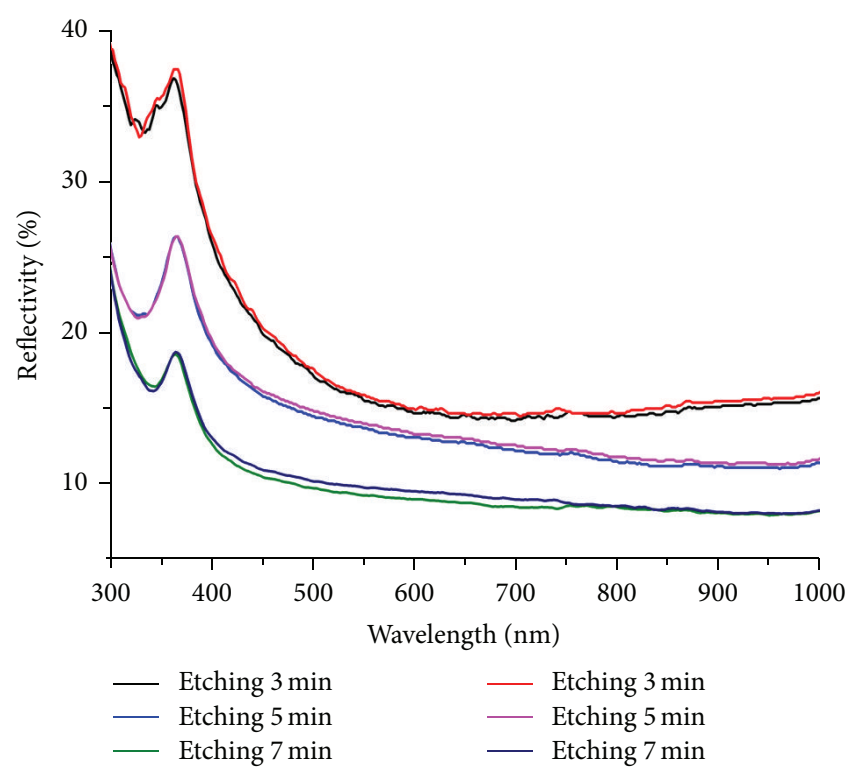

(b)

Figure 5: (a) Specular reflectance curves of flat silicon wafer and Si-hole arrays that etched for $3 \mathrm{~min}, 5 \mathrm{~min}$, and $7 \mathrm{~min}$; (b) homogeneity of the Si-hole arrays substrate.

the Si-hole array (diameter of unetched PS sphere, about $310 \mathrm{~nm}$ ). The morphologies of these Si-hole arrays were characterized by SEM and AFM imaging as shown in Figure 4. Figure 4(a) shows the low-magnified SEM image of the Sihole arrays etched for $3 \mathrm{~min}$. These arrays are long-range ordered following the orientation of spin-coated PS beads. Figure 4(b) shows the high-magnified SEM image of Si-hole arrays. And the inset shows the cross-section view of a Sihole. Figure 4(c) shows the 3D AFM image after etching for $3 \mathrm{~min}$. And Figure 4(d) shows the height profile of the red line marked in Figure 4(c). These AFM images confirmed the morphology of the Si-hole characterized by SEM. Figures $4(\mathrm{e})$ and 4(f) show the SEM images of Si-hole arrays that etched for $5 \mathrm{~min}$ and $7 \mathrm{~min}$, respectively. The insets show the corresponding cross-sections.

Judging from the insets of Figures 4(b), 4(e), and 4(f), the depths of the Si-holes after $3 \mathrm{~min}, 5 \mathrm{~min}$, and $7 \mathrm{~min}$ etching processes were about $122 \mathrm{~nm}, 195 \mathrm{~nm}$, and $285 \mathrm{~nm}$, respectively. All the diameters of the Si-holes are about $300 \mathrm{~nm}$. There is lateral erosion in the Si etching process considering that the corresponding diameters of Cr-masks were $244 \mathrm{~nm}, 219 \mathrm{~nm}$, and $191 \mathrm{~nm}$, respectively. Seen from the insets of Figures 4(e) and 4(f), the sidewalls of the Siholes were nearly triangle. Thus, the Si-hole would not be deepened by extending the etching time due to the lateral erosion. The Si-hole can be deepened if smaller Cr-mask was utilized. Thus, the maximum depth of Si-hole was depending on the diameter of Cr-mask.

3.4. Antireflection Characterization. The patterned Si wafers were utilized for antireflection. These arrays were fabricated with similar diameters but with different depth. The specular reflectance curves of the substrates were shown in Figure 5(a).
The incident light was perpendicular to the substrate surface, and the reflectivity was measured between wavelengths $300 \mathrm{~nm}$ and $1000 \mathrm{~nm}$. The black line represents the reflection curve obtained from a flat $\mathrm{Si}$ wafer. The flat Si wafer exhibits high reflectivity $(>32 \%)$ in both visible and near infrared wavelengths range. All patterned substrates perform much better than the flat silicon substrate. The reflectivity decreased with the depth of the Si-hole arrays. The Si-hole arrays that etched for 7 min exhibit the best antireflection ability $(<9 \%)$, which is about 28 percent of the nonpatterned silicon wafer's reflection.

To investigate the homogeneity of proposed Si-hole arrays, redundancy reflection spectra were measured for each substrate. The redundancy spots were selected far away from the originally measured one on each wafer. These curves were shown in Figure 5(b). The curves coincide to each other well for corresponding etching time, which demonstrated the homogeneity of the patterned antireflection wafer.

The proposed Si-hole based antireflection substrate performs better than the reported colloid-based antireflection coatings $[2,24]$. Moreover, the advantages of the proposed method are the cost-effectiveness, high-throughput, and controllability. And the antireflection performance of proposed structure can be further improved in future research by optimizing the fabrication parameters.

\section{Conclusions}

In summary, a simple, fast, and cost-effective method is developed based on nanosphere lithography in this paper for high-throughput fabrication of wafer-scale nanohole arrays on silicon. These patterned structures perform well in antireflection and have the potential in photovoltaic manufacture. 
The spin-coating technique was introduced herein to confirm the simple, fast, and wafer-scale fabrication. And the fabrication procedure was of high-throughput and cost-effective. It is found that the reflectivity of the substrate decreases with the depth of the Si-hole. And the maximum depth of Sihole is dependent on the diameter of Cr-mask. The Si-hole substrate with $285 \mathrm{~nm}$ depth shows the best antireflection ability ( $<9 \%$ ), which is about 28 percent of the nonpatterned silicon wafer's reflection. The fabricated structures are able to reduce reflectivity at the visible and near infrared wavelengths range. The proposed strategy is attractive for the antireflective substrate fabrication, which have a great potential to improve the solar cell conversion efficiency.

\section{Conflict of Interests}

The authors declare that there is no conflict of interests regarding the publication of this paper.

\section{Acknowledgments}

The authors would like to thank the financial support provided by National Natural Science Foundation of China (91023028) and the Foundation of State Key Laboratory of Transducer Technology (SKT1201).

\section{References}

[1] Y. Tian, C. Hu, Y. Xiong et al., "ZnO pyramidal arrays: novel functionality in antireflection," Journal of Physical Chemistry C, vol. 114, no. 22, pp. 10265-10269, 2010.

[2] C.-H. Sun, W.-L. Min, N. C. Linn, P. Jiang, and B. Jiang, "Templated fabrication of large area subwavelength antireflection gratings on silicon," Applied Physics Letters, vol. 91, no. 23, Article ID 231105, 2007.

[3] C.-H. Sun, W.-L. Min, N. C. Linn, P. Jiang, and B. Jiang, "Largescale assembly of periodic nanostructures with metastable square lattices," Journal of Vacuum Science and Technology B: Microelectronics and Nanometer Structures, vol. 27, no. 3, pp. 1043-1047, 2009.

[4] K.-Q. Peng, X. Wang, L. Li, X.-L. Wu, and S.-T. Lee, "Highperformance silicon nanohole solar cells," Journal of the American Chemical Society, vol. 132, no. 20, pp. 6872-6873, 2010.

[5] Y. Lu and A. Lal, "High-efficiency ordered silicon nano-conicalfrustum array solar cells by self-powered parallel electron lithography," Nano Letters, vol. 10, no. 11, pp. 4651-4656, 2010.

[6] Z. Yu, H. Gao, W. Wu, H. Ge, and S. Y. Chou, "Fabrication of large area subwavelength antireflection structures on $\mathrm{Si}$ using trilayer resist nanoimprint lithography and liftoff," Journal of Vacuum Science and Technology B: Microelectronics and Nanometer Structures, vol. 21, no. 6, pp. 2874-2877, 2003.

[7] N. D. Lam, Y. Kim, K. Kim, and J. Lee, "Influences of InGaP conical frustum nanostructures on the characteristics of GaAs solar cells," Journal of Nanomaterials, vol. 2013, Article ID 785359, 6 pages, 2013.

[8] E. C. Garnett, M. L. Brongersma, Y. Cui, and M. D. McGehee, "Nanowire solar cells," Annual Review of Materials Research, vol. 41, pp. 269-295, 2011.

[9] C.-M. Hsu, S. T. Connor, M. X. Tang, and Y. Cui, "Waferscale silicon nanopillars and nanocones by Langmuir-Blodgett assembly and etching," Applied Physics Letters, vol. 93, no. 13, Article ID 133109, 2008.

[10] N. A. Yahaya, N. Yamada, Y. Kotaki, and T. Nakayama, "Characterization of light absorption in thin-film silicon with periodic nanohole arrays," Optics Express, vol. 21, no. 5, pp. 5924-5930, 2013.

[11] D. Valerie, X. Meng, O. El Daif et al., "Micrometer-thin crystalline-silicon solar cells integrating numerically optimized 2-D photonic crystals," IEEE Journal of Photovoltaics, vol. 4, no. 1, pp. 215-223, 2014.

[12] X. Meng, V. Depauw, G. Gomard et al., "Design, fabrication and optical characterization of photonic crystal assisted thin film monocrystalline-silicon solar cells," Optics Express, vol. 20, supplement 4, pp. A465-A475, 2012.

[13] X. Meng, G. Gomard, O. El Daif et al., "Absorbing photonic crystals for silicon thin-film solar cells: design, fabrication and experimental investigation," Solar Energy Materials and Solar Cells, vol. 95, supplement 1, pp. S32-S38, 2011.

[14] S.-M. Yang, S. G. Jang, D.-G. Choi, S. Kim, and H. K. Yu, "Nanomachining by colloidal lithography," Small, vol. 2, no. 4, pp. 458-475, 2006.

[15] C. Qian, C. Ni, W. Yu et al., "Highly-ordered, 3D petal-like array for surface-enhanced raman scattering," Small, vol. 7, no. 13, pp. 1801-1806, 2011.

[16] S. Zhu and W. Zhou, "Topical review: design, fabrication, and applications of hybrid nanostructured array," Journal of Nanomaterials, vol. 2012, Article ID 206069, 3 pages, 2012.

[17] C. Cong, W. C. Junus, Z. Shen, and T. Yu, "New colloidal lithographic nanopatterns fabricated by combining pre-heating and reactive ion etching," Nanoscale Research Letters, vol. 4, no. 11, pp. 1324-1328, 2009.

[18] H. Qi, O. J. Glembocki, and S. M. Prokes, "Plasmonic properties of vertically aligned nanowire arrays," Journal of Nanomaterials, vol. 2012, Article ID 843402, 7 pages, 2012.

[19] C. Wang, X. Wu, P. Dong et al., "Diversification of nanostructure morphology by modifying angle-resolved heterogeneous shadow mask," Journal of Nanoscience and Nanotechnology, vol. 13, no. 12, pp. 8259-8265, 2013.

[20] M. Giuliani, W. González-Viñas, K. M. Poduska, and A. Yethiraj, "Dynamics of crystal structure formation in spin-coated colloidal films," Journal of Physical Chemistry Letters, vol. 1, no. 9, pp. 1481-1486, 2010.

[21] J. Chen, P. Dong, D. Di et al., "Controllable fabrication of 2D colloidal-crystal films with polystyrene nanospheres of various diameters by spin-coating," Applied Surface Science, vol. 270, pp. 6-15, 2013.

[22] A. R. Madaria, M. Yao, C. Chi et al., "Toward optimized light utilization in nanowire arrays using scalable nanosphere lithography and selected area growth," Nano Letters, vol. 12, no. 6, pp. 2839-2845, 2012.

[23] S. Ye, A. L. Routzahn, and R. L. Carroll, "Fabrication of 3D metal dot arrays by geometrically structured dynamic shadowing lithography," Langmuir, vol. 27, no. 22, pp. 13806-13812, 2011.

[24] N. A. Yahaya, N. Yamada, and T. Nakayama, "Light trapping potential of hexagonal array silicon nanohole structure for solar cell application," Advanced Materials Research, vol. 512-515, pp. 90-96, 2012. 

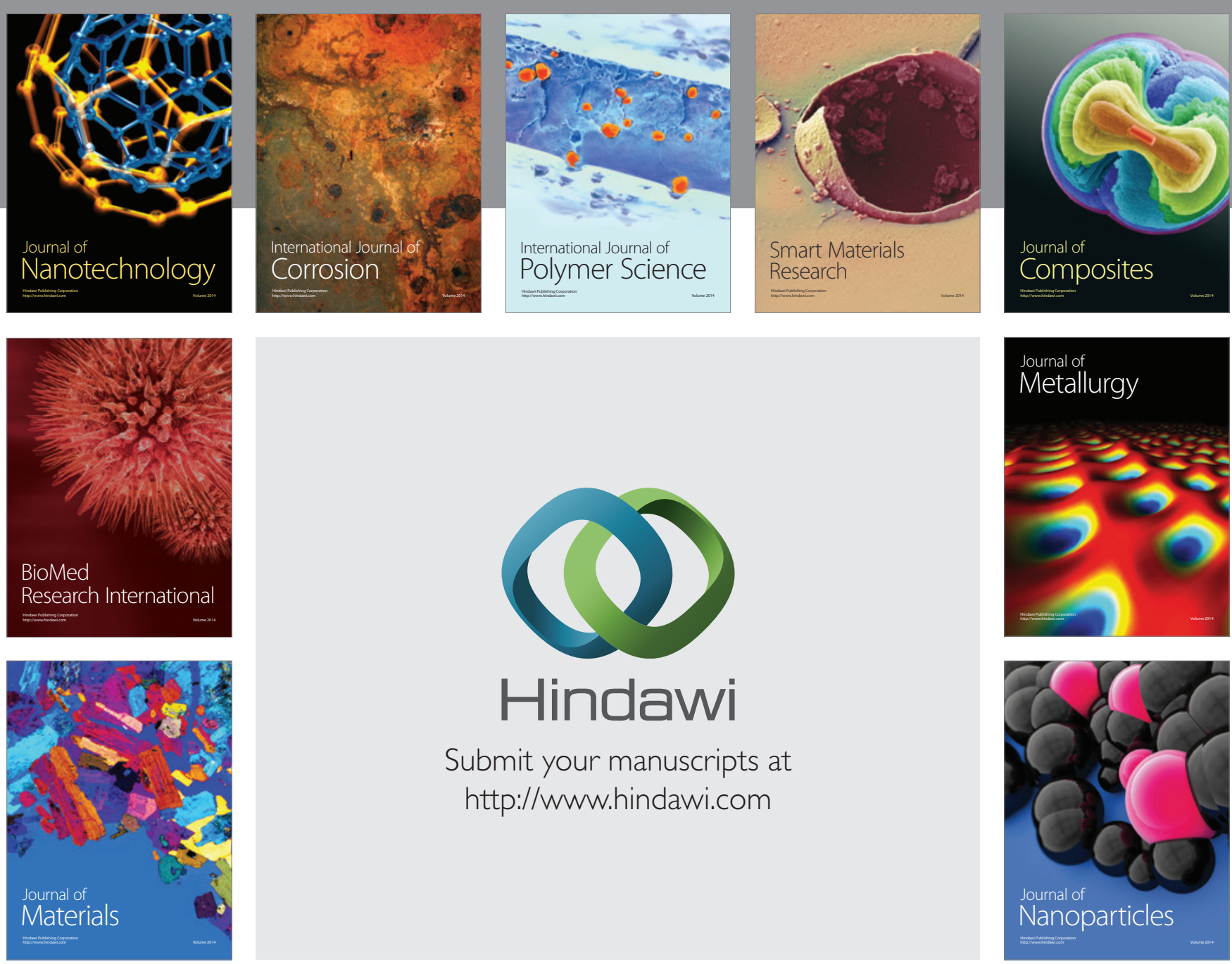

Submit your manuscripts at http://www.hindawi.com
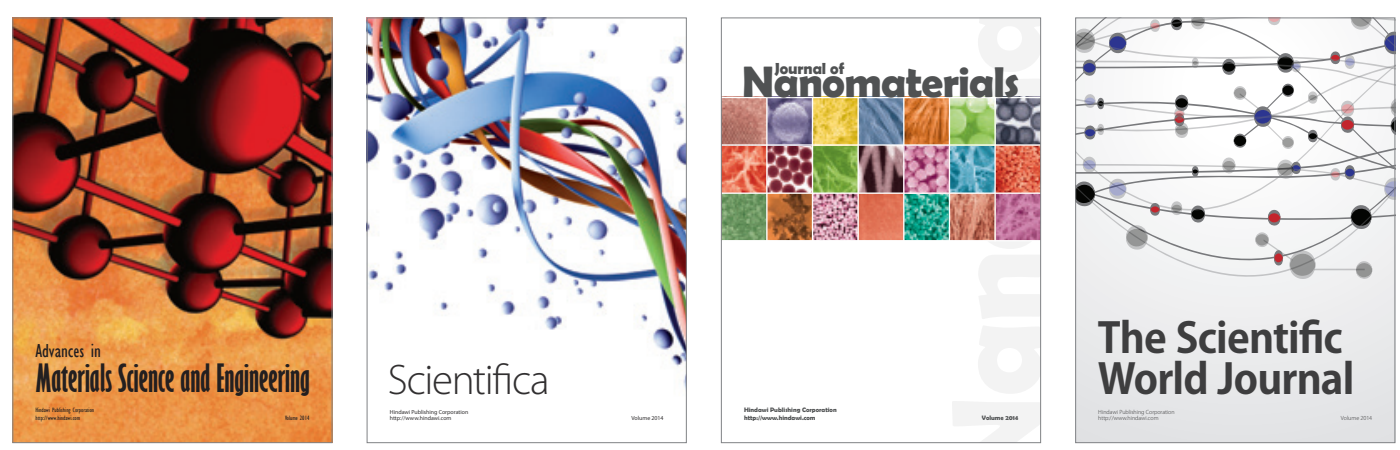

\section{The Scientific World Journal}
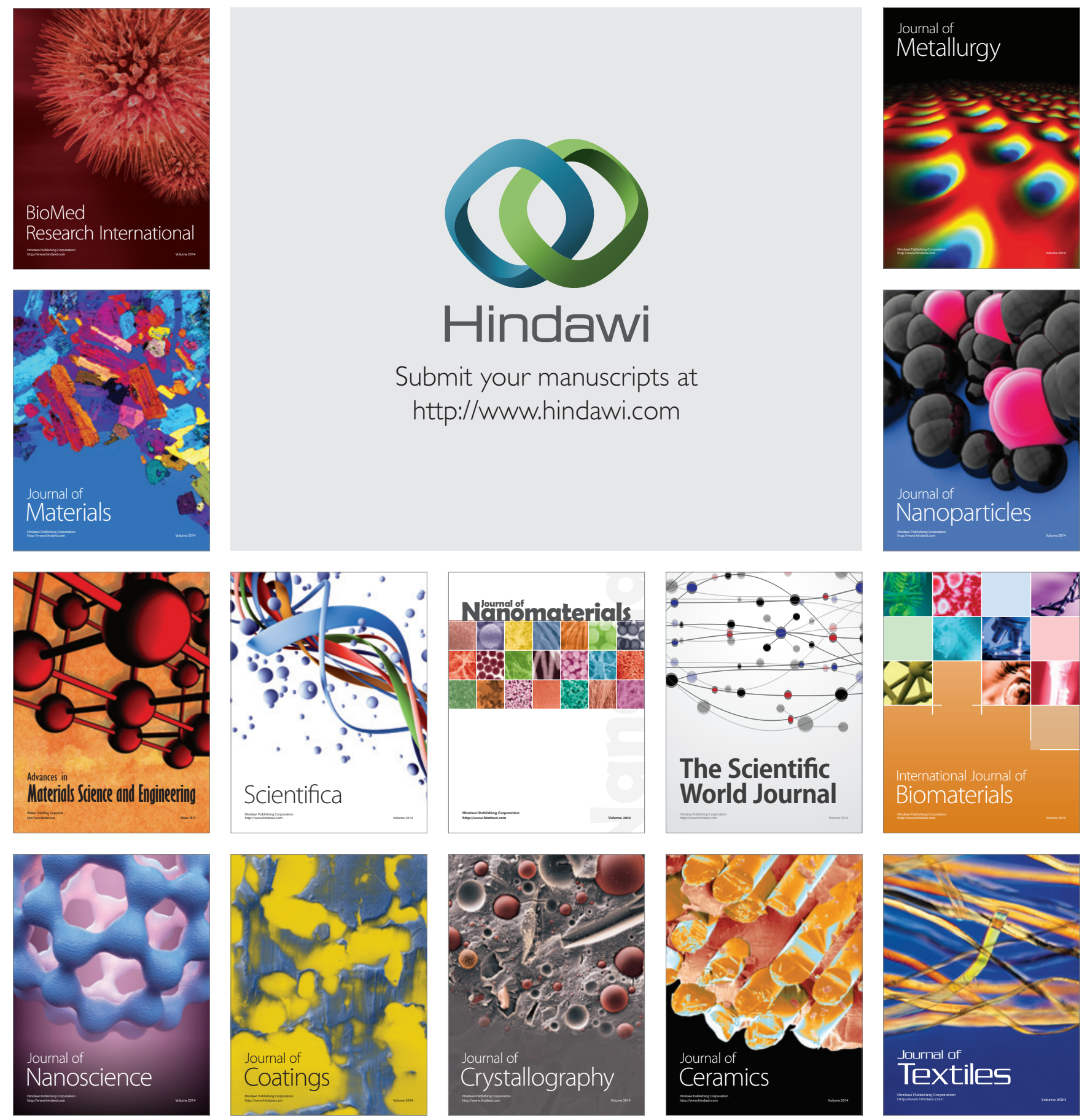